\title{
Nonsquareness and Locally Uniform Nonsquareness in Orlicz-Bochner Function Spaces Endowed with Luxemburg Norm
}

\author{
Shaoqiang Shang, ${ }^{1}$ Yunan $\mathrm{Cui}^{2}{ }^{2}$ and Yongqiang $\mathrm{Fu}^{1}$ \\ ${ }^{1}$ Department of Mathematics, Harbin Institute of Technology, Harbin 150001, China \\ ${ }^{2}$ Department of Mathematics, Harbin University of Science and Technology, Harbin 150080, China
}

Correspondence should be addressed to Shaoqiang Shang, sqshang@163.com

Received 5 July 2010; Accepted 12 February 2011

Academic Editor: Nikolaos Papageorgiou

Copyright (C) 2011 Shaoqiang Shang et al. This is an open access article distributed under the Creative Commons Attribution License, which permits unrestricted use, distribution, and reproduction in any medium, provided the original work is properly cited.

Criteria for nonsquareness and locally uniform nonsquareness of Orlicz-Bochner function spaces equipped with Luxemburg norm are given. We also prove that, in Orlicz-Bochner function spaces generated by locally uniform nonsquare Banach space, nonsquareness and locally uniform nonsquareness are equivalent.

\section{Introduction}

A lot of nonsquareness concepts in Banach spaces are known (see [1]). Nonsquareness are important notions in geometry of Banach space. One of reasons is that these properties are strongly related to the fixed point property (see [2]). The criteria for nonsquareness and locally uniform nonsquareness in the classical Orlicz function spaces have been given in $[3,4]$ already. However, because of the complicated structure of Orlicz-Bochner function spaces equipped with the Luxemburg norm, the criteria for nonsquareness and locally uniform nonsquareness of them have not been found yet. The aim of this paper is to give criteria for nonsquareness and locally uniform nonsquareness of Orlicz-Bochner function spaces equipped with Luxemburg norm.

Let $(X,\|\cdot\|)$ be a real Banach space. $S(X)$ and $B(X)$ denote the unit sphere and unit ball, respectively. Let us recall some geometrical notions concerning nonsquareness. A Banach space $X$ is said to be nonsquare if for any $x, y \in S(X)$ we have $\min \{\|(x+y) / 2\|,\|(x-y) / 2\|\}<$ 1. A Banach space $X$ is said to be uniformly nonsquare if there exists $\delta>0$ such that for any $x, y \in S(X), \min \{\|(x+y) / 2\|,\|(x-y) / 2\|\}<1-\delta$. A Banach space $X$ is said to be locally uniformly nonsquare if for any $x \in S(X)$, there exists $\delta_{x}>0$ such that $\min \{\|(x+y) / 2\|, \|(x-$ $y) / 2 \|\}<1-\delta_{x}$, where $y \in S(X)$. 
Let $R$ be set of real numbers. A function $M: R \rightarrow R^{+}$is called an $N$-function if $M$ is convex, even, $M(0)=0, M(u)>0(u \neq 0)$ and $\lim _{u \rightarrow 0}(M(u) / u)=0$, and $\lim _{u \rightarrow 0}(M(u) / u)$ $=\infty$.

Let $(T, \Sigma, \mu)$ be a nonatomic measurable space. $p$ denotes right derivative of $M$. Moreover, for a given Banach space $(X,\|\cdot\|)$, we denote by $X_{T}$ the set of all strongly $\mu$ measurable function from $T$ to $X$, and for each $u \in X_{T}$, we define the modular of $u$ by

$$
\rho_{M}(u)=\int_{G} M(\|u(t)\|) d t
$$

Put

$$
L_{M}=\left\{u(t) \in X_{T}: \int_{G} M(\|\lambda u(t)\|) d t<\infty \text { for some } \lambda>0\right\}
$$

The linear set $L_{M}$ endowed with the Luxemburg norm

$$
\|u\|=\inf \left\{\lambda>0: \rho_{M}\left(\frac{u}{\lambda}\right) \leq 1\right\}
$$

is a Banach space. We say that an Orlicz function $M$ satisfies condition $\Delta_{2}\left(M \in \Delta_{2}\right)$ if there exist $K>2$ and $u_{0} \geq 0$ such that

$$
M(2 u) \leq K M(u) \quad\left(u \geq u_{0}\right)
$$

First let us recall a known result that will be used in the further part of the paper.

Lemma 1.1 (see [3]). Suppose $M \in \Delta_{2}$. Then

$$
\rho_{M}\left(u_{n}\right) \longrightarrow 0 \Longleftrightarrow\left\|u_{n}\right\| \longrightarrow 0, \quad \rho_{M}\left(u_{n}\right) \longrightarrow 1 \Longleftrightarrow\left\|u_{n}\right\| \longrightarrow 1(n \longrightarrow \infty)
$$

\section{Main Results}

Theorem 2.1. $L_{M}$ is nonsquare if and only if

(a) $M \in \Delta_{2}$;

(b) $X$ is nonsquare.

In order to prove the theorem, we give a lemma.

Lemma 2.2. If $X$ is nonsquare, then for any $x, y \neq 0$, we have

$$
\|x\|+\|y\|-\min \{\|x+y\|,\|x-y\|\}>0
$$


Proof.

Case 1. If $\|x\|<\|y\|$, then

$$
\begin{aligned}
\|x+y\| & \leq\left\|x+\frac{\|x\|}{\|y\|} \cdot y\right\|+\left(1-\frac{\|x\|}{\|y\|}\right) \cdot\|y\| \\
& <\|x\|+\|x\|+\|y\|-\|x\| \\
& =\|x\|+\|y\|
\end{aligned}
$$

or

$$
\|x-y\|<\|x\|+\|y\|
$$

Case 2. If $\|x\| \geq\|y\|$, then

$$
\begin{aligned}
\|x+y\| & \leq\left\|\frac{\|y\|}{\|x\|} \cdot x+y\right\|+\left(\frac{\|x\|}{\|y\|}-1\right) \cdot\|y\| \\
& <\|y\|+\|y\|+\|x\|-\|y\| \\
& =\|x\|+\|y\|
\end{aligned}
$$

or

$$
\|x-y\|<\|x\|+\|y\|
$$

This implies $\|x\|+\|y\|-\min \{\|x+y\|,\|x-y\|\}>0$. This completes the proof.

Proof of Theorem 2.1. (a) Necessity. Suppose that $M \notin \Delta_{2}$, then there exist $u \in S\left(L_{M}\right)$ and $\delta>0$ such that $\rho_{M}(u)=1-\delta<1$. Pick $c>0$ such that $E=\{t \in T:\|u(t)\| \leq c\}$ is not a null set. Since $M \notin \Delta_{2}$, there exist sequence $\left\{r_{n}\right\}_{n=1}^{\infty}$ and disjont subsets $\left\{E_{n}\right\}_{n=1}^{\infty}$ of $E$ such that

$$
r_{n}>2 n c, \quad M\left(\left(1+\frac{1}{n}\right) r_{n}\right)>2^{n} M\left(\left(1+\frac{1}{2 n}\right) r_{n}\right), \quad 2^{n} M\left(\left(1+\frac{1}{2 n}\right) r_{n}\right) \mu E_{n}=2^{-n} \delta .
$$

Therefore, if we define $v=\sum_{n=1}^{\infty} r_{n} \chi_{E_{n}}$, then for any $l>1$, we have

$$
\begin{aligned}
\rho_{M}(l v) & =\sum_{n=1}^{\infty} \rho_{M}\left(\operatorname{lr} r_{n} E_{n}\right) \geq \sum_{n=m}^{\infty} \rho_{M}\left(\left(1+\frac{1}{n}\right) r_{n} X_{E_{n}}\right)>\sum_{n=m}^{\infty} 2^{n} \rho_{M}\left(\left(1+\frac{1}{2 n}\right) r_{n} X_{E_{n}}\right) \\
& \geq \sum_{n=m}^{\infty} 2^{n} M\left(\left(1+\frac{1}{2 n}\right) r_{n}\right) \mu E_{n}=\sum_{n=m}^{\infty} 2^{n} \cdot 2^{-n} \delta=\infty, \\
\rho_{M}(v) & =\sum_{n=1}^{\infty} M\left(r_{n}\right) \mu E_{n}<\sum_{n=1}^{\infty} M\left(r_{n}+c\right) \mu E_{n}<\sum_{n=1}^{\infty} M\left(\left(1+\frac{1}{2 n}\right) r_{n}\right) \mu E_{n}=\delta .
\end{aligned}
$$


This yields

$$
\|v\|=1, \quad \rho_{M}(u \pm v) \leq \rho_{M}(u)+\sum_{n=1}^{\infty} M\left(r_{n}+c\right) \mu E_{n}=1-\delta+\delta=1
$$

Hence, $\|u \pm v\| \leq 1$. But $\|u+v\|+\|u-v\| \leq\|2 u\|=2$, and we deduce that $\|u+v\|=\|u-v\|=1$. Moreover, we have $\|(1 / 2)(u+v)+(1 / 2)(u-v)\|=1$ and $\|(1 / 2)(u+v)-(1 / 2)(u-v)\|\}=1$, a contradiction with nonsquareness of $L_{M}$.

If $(b)$ is not true, then there exist $x, y \in S(X)$ such that $\|x\|=\|y\|=\|(1 / 2)(x+y)\|=$ $\|(1 / 2)(x-y)\|$. Pick $\alpha>0$ such that $\int_{T} M(\alpha) d t=1$. Put

$$
u(t)=\alpha \cdot x \cdot \chi_{T}(t), \quad v(t)=\alpha \cdot y \cdot \chi_{T}(t) .
$$

Then we have

$$
\begin{aligned}
\rho_{M}(u) & =\int_{T} M(\|\alpha x\|) d t=\int_{T} M(\alpha) d t=1 \\
\rho_{M}(v) & =\int_{T} M(\|\alpha y\|) d t=\int_{T} M(\alpha) d t=1 .
\end{aligned}
$$

It is easy to see $u, v \in S\left(L_{M}\right)$. We know that

$$
\frac{u(t)+v(t)}{2}=\alpha \cdot \frac{x+y}{2} \cdot \chi_{T}(t), \quad \frac{u(t)-v(t)}{2}=\alpha \cdot \frac{x-y}{2} \cdot \chi_{T}(t) .
$$

Hence, we have

$$
\begin{aligned}
& \rho_{M}\left(\frac{u+v}{2}\right)=\int_{T} M\left(\left\|\alpha \cdot \frac{x+y}{2}\right\|\right) d t=\int_{T} M(\alpha) d t=1, \\
& \rho_{M}\left(\frac{u-v}{2}\right)=\int_{T} M\left(\left\|\alpha \cdot \frac{x-y}{2}\right\|\right) d t=\int_{T} M(\alpha) d t=1 .
\end{aligned}
$$

It is easy to see $(1 / 2)(u+v),(1 / 2)(u-v) \in S\left(L_{M}\right)$, a contradiction!

Sufficiency. Suppose that there exists $u, v \in S\left(L_{M}\right)$ such that

$$
\|u\|=\|v\|=\left\|\frac{1}{2}(u+v)\right\|=\left\|\frac{1}{2}(u-v)\right\|=1 .
$$

We will derive a contradiction for each of the following two cases. 
Case 1. $\mu(\{t \in T:\|u(t)\| \neq 0\} \cap\{t \in T:\|v(t)\| \neq 0\})=0$. Let $G=\{t \in T:\|u(t)\| \neq 0\}$. Hence, we have

$$
\begin{aligned}
\frac{1}{2} \rho_{M}(u)+\frac{1}{2} \rho_{M}(v) & =\frac{1}{2} \int_{G} M(\|u(t)\|) d t+\frac{1}{2} \int_{T \backslash G} M(\|v(t)\|) d t \\
& =\frac{1}{2} \int_{G} M(\|u(t)+v(t)\|) d t+\frac{1}{2} \int_{T \backslash G} M(\|u(t)+v(t)\|) d t \\
& >\int_{G} M\left(\frac{1}{2}\|u(t)+v(t)\|\right) d t+\int_{T \backslash G} M\left(\frac{1}{2}\|u(t)+v(t)\|\right) d t \\
& =\int_{T} M\left(\frac{1}{2}\|u(t)+v(t)\|\right) d t \\
& =\rho_{M}\left(\frac{1}{2}(u+v)\right)
\end{aligned}
$$

Since $M \in \Delta_{2}$, we have $\rho_{M}(u)=\rho_{M}(v)=1$. Hence, $\rho_{M}((u+v) / 2)<1$. This implies $\|(u+$ $v) / 2 \|<1$, a contradiction!

Case 2. $\mu(\{t \in T:\|u(t)\| \neq 0\} \cap\{t \in T:\|v(t)\| \neq 0\})>0$. By Lemma 2.2, without loss of generality, we may assume that there exists $T_{1} \subset\{t \in T:\|u(t)\| \neq 0\} \cap\{t \in T:\|v(t)\| \neq 0\}$ such that $\|u(t)\|+\|v(t)\|>\|u(t)+v(t)\|, t \in T_{1}$ and $\mu T_{1}>0$. Therefore,

$$
\begin{aligned}
\frac{1}{2} \rho_{M}(u)+\frac{1}{2} \rho_{M}(v) & =\frac{1}{2} \int_{T} M(\|u(t)\|) d t+\frac{1}{2} \int_{T} M(\|v(t)\|) d t \\
& =\int_{T} \frac{1}{2} M(\|u(t)\|)+\frac{1}{2} M(\|v(t)\|) d t \\
& \geq \int_{T_{1}} M\left(\frac{1}{2}\|u(t)\|+\frac{1}{2}\|v(t)\|\right) d t+\int_{T \backslash T_{1}} M\left(\frac{1}{2}\|u(t)\|+\frac{1}{2}\|v(t)\|\right) d t \\
& >\int_{T_{1}} M\left(\frac{1}{2}\|u(t)+v(t)\|\right) d t+\int_{T \backslash T_{1}} M\left(\frac{1}{2}\|u(t)+v(t)\|\right) d t \\
& =\rho_{M}\left(\frac{u+v}{2}\right) .
\end{aligned}
$$

Since $M \in \Delta_{2}$, we have $\rho_{M}(u)=\rho_{M}(v)=1$. Hence, $\rho_{M}((u+v) / 2)<1$. This implies $\|(u+v) / 2\|<1$, a contradiction!

Theorem 2.3. $L_{M}$ is locally uniformly nonsquare if and only if

(a) $M \in \Delta_{2}$;

(b) $\mathrm{X}$ is locally uniformly nonsquare. 
In order to prove the theorem, we give a lemma.

Lemma 2.4. If $X$ is locally uniformly nonsquare, then

(a) For any $x \neq 0, r_{1} \geq r_{2}>0$, we have

$$
\inf _{y \neq 0}\left\{\|x\|+\|y\|-\min \{\|x+y\|,\|x-y\|\}: x \in X, r_{2} \leq\|y\| \leq r_{1}\right\}>0
$$

(b) If $x_{n} \rightarrow x$, then $\lim _{n \rightarrow \infty} \delta\left(x_{n}\right)=\delta(x)$, where

$$
\delta(x)=\inf _{y \neq 0}\left\{\|x\|+\|y\|-\min \{\|x+y\|,\|x-y\|\}: x \in X, r_{2} \leq\|y\| \leq r_{1}\right\} .
$$

Proof. (a) Since $X$ is locally uniformly nonsquare, we have $\eta_{x}>0$ and $\eta_{\lambda x}=\lambda \eta_{x}$, where $\lambda>0$ and

$$
\eta_{x}=\inf _{y}\{\|x\|+\|y\|-\min \{\|x+y\|,\|x-y\|\}:\|x\|=\|y\|>0\}
$$

In fact, since $X$ is locally uniformly nonsquare, we have

$$
\begin{aligned}
\eta_{x} & =\inf _{y}\{\|x\|+\|y\|-\min \{\|x+y\|,\|x-y\|\}:\|x\|=\|y\|>0\} \\
& =\|x\| \cdot \inf _{y}\left\{2-\min \left\{\left\|\frac{x}{\|x\|}+\frac{y}{\|y\|}\right\|,\left\|\frac{x}{\|x\|}-\frac{y}{\|y\|}\right\|\right\}:\|x\|=\|y\|>0\right\}>0, \\
\eta_{\lambda x} & =\inf _{y}\{\|\lambda x\|+\|y\|-\min \{\|\lambda x+y\|,\|\lambda x-y\|\}:\|\lambda x\|=\|y\|>0\} \\
& =\lambda \cdot \inf _{y}\left\{\|x\|+\left\|\frac{1}{\lambda} y\right\|-\min \left\{\left\|x+\frac{1}{\lambda} y\right\|,\left\|x-\frac{1}{\lambda} y\right\|\right\}:\|x\|=\left\|\frac{1}{\lambda} y\right\|>0\right\} \\
& =\lambda \cdot \inf _{y}\{\|x\|+\|y\|-\min \{\|x+y\|,\|x-y\|\}:\|x\|=\|y\|>0\} \\
& =\lambda \cdot \eta_{x} .
\end{aligned}
$$


Case 1. If $\|x\| \geq\|y\|$, then

$$
\begin{aligned}
\|x+y\| & \leq\left\|\left(1-\frac{\|y\|}{\|x\|}\right) x+y+\frac{\|y\|}{\|x\|} x\right\| \\
& \leq\left\|\left(1-\frac{\|y\|}{\|x\|}\right) x\right\|+\left\|y+\frac{\|y\|}{\|x\|} x\right\| \\
& \leq\|x\|-\|y\|+\|y\|+\|y\|-\eta_{(\|y\| /\|x\|) x} \\
& \leq\|x\|+\|y\|-\eta_{\left(r_{2} /\|x\|\right) x}
\end{aligned}
$$

or

$$
\|x-y\| \leq\|x\|+\|y\|-\eta_{\left(r_{2} /\|x\|\right) x} .
$$

Case 2. If $\|x\|<\|y\|$, then

$$
\begin{aligned}
\|x+y\| & \leq\left\|\frac{\|x\|}{\|y\|} \cdot y+x\right\|+\left(1-\frac{\|x\|}{\|y\|}\right) \cdot\|y\| \\
& \leq\|x\|+\|x\|-\eta_{x}+\|y\|-\|x\| \\
& =\|x\|+\|y\|-\eta_{x} \\
& \leq\|x\|+\|y\|-\eta_{x}
\end{aligned}
$$

or

$$
\|x-y\| \leq\|x\|+\|y\|-\eta_{x}
$$

Therefore, we get, the following inequality

$$
\inf _{y}\{\|x\|+\|y\|-\min \{\|x+y\|,\|x-y\|\}: x \in X\} \geq \min \left\{\eta_{\left(r_{2} /\|x\|\right) x}, \eta_{x}\right\}>0
$$

holds.

(b1) Suppose that $\lim \sup _{n \rightarrow \infty} \delta\left(x_{n}\right)>\delta(x)$, where $x_{n} \rightarrow x(n \rightarrow \infty)$. Then there exist $a>0$ and subsequence $\{n\}$ of $\{n\}$, such that $\delta\left(x_{n}\right)-\delta(x) \geq a$. By definition of $\delta(x)$, there exist $y_{0} \in X$ such that

$$
\|x\|+\left\|y_{0}\right\|-\min \left\{\left\|x+y_{0}\right\|,\left\|x-y_{0}\right\|\right\}<\delta(x)+\frac{a}{8}, \quad r_{1} \leq\left\|y_{0}\right\| \leq r_{2}
$$

We will derive a contradiction for each of the following two cases. 
Case 1. $\left\|x+y_{0}\right\|=\left\|x-y_{0}\right\|$. Since $x_{n} \rightarrow x(n \rightarrow \infty)$, there exists $n_{0}$ such that $\left\|x_{n_{0}}-x\right\|<a / 8$. Therefore,

$$
\begin{aligned}
\left\|x_{n_{0}}\right\|+\left\|y_{0}\right\|-\left\|x_{n_{0}}+y_{0}\right\| & \leq\|x\|+\left\|x_{n_{0}}-x\right\|+\left\|y_{0}\right\|-\left\|x_{n_{0}}+y_{0}\right\| \\
& \leq\|x\|+\left\|x_{n_{0}}-x\right\|+\left\|y_{0}\right\|-\left(\left\|x+y_{0}\right\|-\left\|x_{n_{0}}-x\right\|\right) \\
& =\|x\|+\left\|y_{0}\right\|-\left\|x+y_{0}\right\|+2\left\|x_{n_{0}}-x\right\| \\
& \leq \delta(x)+\frac{a}{8}+2\left\|x_{n_{0}}-x\right\| \\
& <\delta(x)+\frac{a}{8}+2 \cdot \frac{a}{8} \\
& =\delta(x)+\frac{3}{8} a .
\end{aligned}
$$

This implies $\delta\left(x_{n_{0}}\right) \leq \delta(x)+(3 / 8) a$, a contradiction!

Case 2. $\left\|x-y_{0}\right\| \neq\left\|x+y_{0}\right\|$. Without loss of generality, we may assume $\left\|x-y_{0}\right\|>\left\|x+y_{0}\right\|+r$, where $r>0$. Since $x_{n} \rightarrow x(n \rightarrow \infty)$, there exists $n_{0}$ such that $\left\|x_{n_{0}}-x\right\|<\min \{(1 / 8) a,(1 / 8) r\}$. Therefore, we have

$$
\begin{aligned}
\left\|x_{n_{0}}-y_{0}\right\| & =\left\|x-y_{0}+x_{n_{0}}-x\right\| \\
& \geq\left\|x-y_{0}\right\|-\left\|x_{n_{0}}-x\right\| \\
& \geq\left\|x-y_{0}\right\|-\frac{1}{8} r, \\
\left\|x_{n_{0}}+y_{0}\right\| & =\left\|x+y_{0}+x_{n_{0}}-x\right\| \\
& \leq\left\|x+y_{0}\right\|+\left\|x_{n_{0}}-x\right\| \\
& \leq\left\|x+y_{0}\right\|+\frac{1}{8} r .
\end{aligned}
$$

This implies

$$
\left\|x_{n_{0}}-y_{0}\right\| \geq\left\|x-y_{0}\right\|-\frac{1}{8} r \geq\left\|x+y_{0}\right\|+r-\frac{1}{8} r \geq\left\|x+y_{0}\right\|+\frac{1}{8} r \geq\left\|x_{n_{0}}+y_{0}\right\| .
$$

Similarly, we have

$$
\left\|x_{n_{0}}\right\|+\left\|y_{0}\right\|-\left\|x_{n_{0}}+y_{0}\right\| \leq \delta(x)+\frac{3}{8} a
$$

Therefore, we have

$$
\left\|x_{n_{0}}\right\|+\left\|y_{0}\right\|-\min \left\{\left\|x_{n_{0}}+y_{0}\right\|,\left\|x_{n_{0}}-y_{0}\right\|\right\} \leq \delta(x)+\frac{3}{8} a
$$

This implies $\delta\left(x_{n_{0}}\right) \leq \delta(x)+(3 / 8) a$, a contradiction! Hence, $\lim \sup _{n \rightarrow \infty} \delta\left(x_{n}\right) \leq \delta(x)$. 
(b2) Suppose that $\lim \inf _{n \rightarrow \infty} \delta\left(x_{n}\right)<\delta(x)$, where $x_{n} \rightarrow x(n \rightarrow \infty)$. Then there exist $b>0$ and subsequence $\{n\}$ of $\{n\}$, such that $\delta(x)-\delta\left(x_{n}\right) \geq b$. Since $x_{n} \rightarrow x(n \rightarrow \infty)$, then there exist $n_{0} \in N$ such that $\left\|x_{n_{0}}-x_{n}\right\|<(1 / 8) b$, whenever $n \geq n_{0}$. By definition of $\delta\left(x_{n_{0}}\right)$, there exist $y_{0} \in X$ such that

$$
\left\|x_{n_{0}}\right\|+\left\|y_{0}\right\|-\min \left\{\left\|x_{n_{0}}+y_{0}\right\|,\left\|x_{n_{0}}-y_{0}\right\|\right\}<\delta\left(x_{n_{0}}\right)+\frac{b}{8}, \quad r_{2} \leq\left\|y_{0}\right\| \leq r_{1}
$$

Therefore, we have

$$
\begin{aligned}
\left\|x_{n}\right\| & +\left\|y_{0}\right\|-\min \left\{\left\|x_{n}+y_{0}\right\|,\left\|x_{n}-y_{0}\right\|\right\} \\
& =\left\|x_{n_{0}}-x_{n_{0}}+x_{n}\right\|+\left\|y_{0}\right\|-\min \left\{\left\|x_{n_{0}}-x_{n_{0}}+x_{n}+y_{0}\right\|,\left\|x_{n_{0}}-x_{n_{0}}+x_{n}-y_{0}\right\|\right\} \\
& \leq\left\|x_{n_{0}}\right\|+\frac{1}{8} b+\left\|y_{0}\right\|-\min \left\{\left\|x_{n_{0}}+y_{0}\right\|,\left\|x_{n_{0}}-y_{0}\right\|\right\}+\frac{1}{8} b \\
& =\left\|x_{n_{0}}\right\|+\left\|y_{0}\right\|-\min \left\{\left\|x_{n_{0}}+y_{0}\right\|,\left\|x_{n_{0}}-y_{0}\right\|\right\}+\frac{1}{4} b \\
& <\delta\left(x_{n_{0}}\right)+\frac{1}{8} b+\frac{1}{4} b \\
& <\delta\left(x_{n_{0}}\right)+\frac{3}{8} b
\end{aligned}
$$

whenever $n \geq n_{0}$. Since $x_{n} \rightarrow x(n \rightarrow \infty)$, there exists $n_{1}>n_{0}$ such that $\left|\eta(x)-\eta\left(x_{n_{1}}\right)\right|<$ $(1 / 8) b$, where

$$
\eta(x)=\|x\|+\left\|y_{0}\right\|-\min \left\{\left\|x+y_{0}\right\|,\left\|x-y_{0}\right\|\right\}
$$

Hence, we have

$$
\eta\left(x_{n_{1}}\right)>\eta(x)-\frac{1}{8} b \geq \delta(x)-\frac{1}{8} b \geq \delta\left(x_{n_{0}}\right)+b-\frac{1}{8} b=\delta\left(x_{n_{0}}\right)+\frac{7}{8} b .
$$

This implies

$$
\left\|x_{n_{1}}\right\|+\left\|y_{0}\right\|-\min \left\{\left\|x_{n_{1}}+y_{0}\right\|,\left\|x_{n_{1}}-y_{0}\right\|\right\} \geq \delta\left(x_{n_{0}}\right)+\frac{7}{8} b
$$

which contradict (2.32). Hence, $\lim \inf _{n \rightarrow \infty} \delta\left(x_{n}\right) \geq \delta(x)$.

Combing (b1) with (b2), we get $\lim _{n \rightarrow \infty} \delta\left(x_{n}\right)=\delta(x)$. This completes the proof. 
Proof of Theorem 2.3. Necessity. By Theorem 2.1, $M \in \Delta_{2}$. If (b) is not true, then there exist $x \in S(X),\left\{y_{n}\right\}_{n=1}^{\infty} \subset S(X)$ such that $\left\|(1 / 2)\left(x+y_{n}\right)\right\| \rightarrow 1$ and $\left\|(1 / 2)\left(x-y_{n}\right)\right\| \rightarrow 1$ as $n \rightarrow \infty$. Pick $\alpha>0$ such that $\int_{T} M(\alpha) d t=1$. Put

$$
u(t)=\alpha \cdot x \cdot \chi_{T}(t), v_{n}(t)=\alpha \cdot y_{n} \cdot \chi_{T}(t)
$$

Then we have

$$
\begin{gathered}
\rho_{M}(u)=\int_{T} M(\|\alpha x\|) d t=\int_{T} M(\alpha) d t=1, \\
\rho_{M}\left(v_{n}\right)=\int_{T} M\left(\left\|\alpha y_{n}\right\|\right) d t=\int_{T} M(\alpha) d t=1 .
\end{gathered}
$$

It is easy to see $u, v_{n} \in S\left(L_{M}\right)$. We know that

$$
\frac{u(t)+v_{n}(t)}{2}=\alpha \cdot \frac{x+y_{n}}{2} \cdot \chi_{T}(t), \quad \frac{u(t)-v_{n}(t)}{2}=\alpha \cdot \frac{x-y_{n}}{2} \cdot \chi_{T}(t)
$$

Moreover, we have $M\left(\alpha \cdot\left\|\left(x+y_{n}\right) / 2\right\|\right) \leq M(\alpha), M\left(\alpha \cdot\left\|\left(x-y_{n}\right) / 2\right\|\right) \leq M(\alpha)$. By the dominated convergence theorem, we have

$$
\begin{aligned}
& \lim _{n \rightarrow \infty} \int_{T} M\left(\alpha \cdot\left\|\frac{x+y_{n}}{2}\right\|\right) d t=\int_{T} \lim _{n \rightarrow \infty} M\left(\alpha \cdot\left\|\frac{x+y_{n}}{2}\right\|\right) d t=\int_{T} M(\alpha) d t=1, \\
& \lim _{n \rightarrow \infty} \int_{T} M\left(\alpha \cdot\left\|\frac{x-y_{n}}{2}\right\|\right) d t=\int_{T} \lim _{n \rightarrow \infty} M\left(\alpha \cdot\left\|\frac{x-y_{n}}{2}\right\|\right) d t=\int_{T} M(\alpha) d t=1 .
\end{aligned}
$$

It is easy to see $\rho_{M}\left((1 / 2)\left(u+v_{n}\right)\right) \rightarrow 1, \rho_{M}\left((1 / 2)\left(u-v_{n}\right)\right) \rightarrow 1$ as $n \rightarrow \infty$. By Lemma 1.1, we have $\left\|(1 / 2)\left(u+v_{n}\right)\right\| \rightarrow 1$ and $\left\|(1 / 2)\left(u-v_{n}\right)\right\| \rightarrow 1$ as $n \rightarrow \infty$, a contradiction with locally uniform nonsquareness of $L_{M}$.

Sufficiency. Suppose that there exist $u \in S\left(L_{M}\right),\left\{v_{n}\right\}_{n=1}^{\infty} \subset S\left(L_{M}\right)$ such that $\|(1 / 2)(u+$ $\left.v_{n}\right)\|\rightarrow 1\|,(1 / 2)\left(u-v_{n}\right) \| \rightarrow 1$ as $n \rightarrow \infty$. We will derive a contradiction for each of the following two cases.

Case 1. There exist $\varepsilon_{0}>0, \sigma_{0}>0$ such that $\mu G_{n}>\varepsilon_{0}$, where $G_{n}=\left\{t \in T:\left\|v_{n}(t)\right\| \geq \sigma_{0}\right\}$. Put

$$
H_{n}=\left\{t \in T: \sigma_{0} \leq\left\|v_{n}(t)\right\| \leq M^{-1}\left(\frac{4}{\varepsilon_{0}}\right)\right\} .
$$

We have

$$
1=\int_{T} M\left(\left\|v_{n}(t)\right\|\right) d t \geq \int_{G_{n} \backslash H_{n}} M\left(\left\|v_{n}(t)\right\|\right) d t \geq \frac{4}{\varepsilon_{0}} \cdot \mu\left(G_{n} \backslash H_{n}\right) .
$$


Journal of Inequalities and Applications

This implies $\mu\left(G_{n} \backslash H_{n}\right) \leq(1 / 4) \varepsilon_{0}$. Hence, $\mu H_{n}>(1 / 2) \varepsilon_{0}$. We define a function

$$
\eta(t)=\inf _{y \neq 0}\left\{\|u(t)\|+\|y\|-\min \{\|u(t)+y\|,\|u(t)-y\|\}: \sigma_{0} \leq\|y\| \leq M^{-1}\left(\frac{4}{\varepsilon_{0}}\right)\right\}
$$

on $T_{0}$, where $T_{0}=\{t \in T:\|u(t)\| \neq 0\}$. By Lemma 2.4, we have $\eta(t)>0 \mu$-a.e on $T_{0}$. Let $h_{n}(t) \rightarrow u(t) \mu$-a.e on $T_{0}$, where $h_{n}$ is simple function. Hence,

$$
\eta_{n}(t)=\inf _{y \neq 0}\left\{\left\|h_{n}(t)\right\|+\|y\|-\min \left\{\left\|h_{n}(t)+y\right\|,\left\|h_{n}(t)-y\right\|\right\}: \sigma_{0} \leq\|y\| \leq M^{-1}\left(\frac{4}{\varepsilon_{0}}\right)\right\}
$$

is $\mu$-measurable. By Lemma 2.4, we have $\eta_{n}(t) \rightarrow \eta(t) \mu$-a.e on $T_{0}$. Then $\eta(t)$ is $\mu$-measurable. Using

$$
T \bigcup_{i=1}^{\infty}\left\{t \in T_{0}: \frac{1}{i+1}<\eta(t) \leq \frac{1}{i}\right\}
$$

we get that there exists $\eta_{0}>0$ such that $\mu H<(1 / 8) \varepsilon_{0}$, where

$$
H=\left\{t \in T_{0}: \eta(t) \leq 2 \eta_{0}\right\}
$$

Let $E_{n}=H_{n} \backslash H, E_{n}^{1}=\left(H_{n} \cap\{t \in T:\|u(t)\| \neq 0\}\right) \backslash H, E_{n}^{2}=\left(H_{n} \cap\{t \in T:\|u(t)\|=0\}\right) \backslash H$. It is easy to see $E_{n}=E_{n}^{1} \cup E_{n}^{2}, E_{n}^{1} \cap E_{n}^{2}=\phi$ and $\mu E_{n} \geq(3 / 8) \varepsilon_{0}$. If $t \in E_{n}^{1}$, by Lemma 2.4, we have

$$
\|u(t)\|+\left\|v_{n}(t)\right\|-\min \left\{\left\|u(t)+v_{n}(t)\right\|,\left\|u(t)-v_{n}(t)\right\|\right\} \geq \eta(t) \geq 2 \eta_{0} .
$$

Without loss of generality, we may assume that there exists $F_{n}^{1} \subset E_{n}^{1}$ such that

$$
\mu F_{n}^{1} \geq \frac{1}{2} \mu E_{n}^{1}, \quad\|u(t)\|+\left\|v_{n}(t)\right\|-\left\|u(t)+v_{n}(t)\right\| \geq 2 \eta_{0} t \in F_{n}^{1} .
$$

Moreover, for any $u \geq v>0$, we have

$$
\begin{aligned}
& \frac{1}{2} M(u)-M\left(\frac{1}{2} u\right)-\left[\frac{1}{2} M(v)-M\left(\frac{1}{2} v\right)\right] \\
&= \frac{1}{2} \int_{0}^{u} p(t) d t-\int_{0}^{(1 / 2) u} p(t) d t-\frac{1}{2} \int_{0}^{v} p(t) d t+\int_{0}^{(1 / 2) v} p(t) d t \\
&= {\left[\frac{1}{2} \int_{0}^{u} p(t) d t-\frac{1}{2} \int_{0}^{v} p(t) d t\right]-\left[\int_{0}^{(1 / 2) u} p(t) d t-\int_{0}^{(1 / 2) v} p(t) d t\right] } \\
&= \frac{1}{2} \int_{v}^{u} p(t) d t-\int_{(1 / 2) v}^{(1 / 2) u} p(t) d t \\
& \geq \int_{v}^{(1 / 2) u+(1 / 2) v} p(t) d t-\int_{(1 / 2) v}^{(1 / 2) u} p(t) d t \geq 0 .
\end{aligned}
$$


Hence, if $t \in E_{n}^{2}$, then

$$
\frac{1}{2} M\left(\left\|v_{n}(t)\right\|\right)+\frac{1}{2} M\left(\left\|v_{n}(t)\right\|\right) \geq \frac{1}{2} M\left(\sigma_{0}\right)-M\left(\frac{\sigma_{0}}{2}\right)>0
$$

Let $F_{n}=F_{n}^{1} \cup E_{n}^{2}$. Then $\mu F_{n} \geq(1 / 8) \varepsilon_{0}$. Therefore,

$$
\begin{aligned}
& \frac{1}{2} \rho_{M}(u)+\frac{1}{2} \rho_{M}\left(v_{n}\right)-\rho_{M}\left(\frac{u+v_{n}}{2}\right) \\
&=\frac{1}{2} \int_{T} M(\|u(t)\|) d t+\frac{1}{2} \int_{T} M\left(\left\|v_{n}(t)\right\|\right) d t-\int_{T} M\left(\frac{\left\|u(t)+v_{n}(t)\right\|}{2}\right) d t \\
&=\int_{T}\left[\frac{1}{2} M(\|u(t)\|)+\frac{1}{2} M\left(\left\|v_{n}(t)\right\|\right)-M\left(\frac{\left\|u(t)+v_{n}(t)\right\|}{2}\right)\right] d t \\
& \geq \int_{F_{n}^{1}}\left[\frac{1}{2} M(\|u(t)\|)+\frac{1}{2} M\left(\left\|v_{n}(t)\right\|\right)-M\left(\frac{\left\|u(t)+v_{n}(t)\right\|}{2}\right)\right] d t \\
&+\int_{E_{n}^{2}}\left[\frac{1}{2} M(\|u(t)\|)+\frac{1}{2} M\left(\left\|v_{n}(t)\right\|\right)-M\left(\frac{\left\|u(t)+v_{n}(t)\right\|}{2}\right)\right] d t \\
& \geq \int_{F_{n}^{1}}\left[M\left(\frac{1}{2}\|u(t)\|+\frac{1}{2}\left\|v_{n}(t)\right\|\right)-M\left(\frac{\left\|u(t)+v_{n}(t)\right\|}{2}\right)\right] d t \\
&+\int_{E_{n}^{2}}\left[\frac{1}{2} M(\|u(t)\|)+\frac{1}{2} M\left(\left\|v_{n}(t)\right\|\right)-M\left(\frac{\left\|u(t)+v_{n}(t)\right\|}{2}\right)\right] d t \\
& \geq \int_{F_{n}^{1}}\left[M\left(\frac{\left\|u(t)+v_{n}(t)\right\|}{2}+\eta_{0}\right)-M\left(\frac{\left\|u(t)+v_{n}(t)\right\|}{2}\right)\right] d t \\
&+\int_{E_{n}^{2}}\left[\frac{1}{2} M\left(\left\|v_{n}(t)\right\|\right)-M\left(\frac{\left\|v_{n}(t)\right\|}{2}\right)\right] d t \\
& \geq \int_{F_{n}^{1}} M\left(\eta_{0}\right) d t+\int_{E_{n}^{2}}\left[\frac{1}{2} M\left(\sigma_{0}\right)-M\left(\frac{\sigma_{0}}{2}\right)\right] d t \\
& \geq \int_{F_{n}} \min \left\{M\left(\eta_{0}\right), \frac{1}{2} M\left(\sigma_{0}\right)-M\left(\frac{\sigma_{0}}{2}\right)\right\} d t \\
&= \min \left\{M\left(\eta_{0}\right), \frac{1}{2} M\left(\sigma_{0}\right)-M\left(\frac{\sigma_{0}}{2}\right)\right\} \cdot \mu F_{n} \\
& \geq\left.M\left(\eta_{0}\right), \frac{1}{2} M\left(\sigma_{0}\right)-M\left(\frac{\sigma_{0}}{2}\right)\right\} \cdot \frac{1}{8} \varepsilon_{0} .
\end{aligned}
$$

By Lemma 1.1, we have $\rho_{M}(u)=\rho_{M}\left(v_{n}\right)=1, \rho_{M}\left(\left(u+v_{n}\right) / 2\right) \rightarrow 1$ as $n \rightarrow \infty$. This is in contradiction with $(1 / 2) \rho_{M}(u)+(1 / 2) \rho_{M}\left(v_{n}\right)-\rho_{M}\left(\left(u+v_{n}\right) / 2\right) \geq \min \left\{M\left(\eta_{0}\right),(1 / 2) M\left(\sigma_{0}\right)-\right.$ $\left.M\left(\sigma_{0} / 2\right)\right\} \cdot(1 / 8) \varepsilon_{0}$. 
Case 2. For any $\varepsilon>0, \sigma>0$, there exists $N$ such that $\mu\left\{t \in T:\left\|v_{n}(t)\right\| \geq \sigma\right\}<\varepsilon$ whenever $n>N$. By the Riesz theorem, without loss of generality, we may assume that $v_{n}(t) \rightarrow 0 \mu-$ a.e on $T$. Using

$$
\{t \in T:\|u(t)\| \neq 0\} \supset \bigcup_{n=1}^{\infty}\left\{t \in T: \frac{1}{n+1}<\|u(t)\| \leq \frac{1}{n}\right\}
$$

we get that there exist $r>0$ such that $\mu T_{1}<(1 / 8) \mu T_{0}$, where

$$
T_{1}=\{t \in T: 0<\|u(t)\|<d\}, \quad T_{0}=\{t \in T:\|u(t)\| \neq 0\} .
$$

Since $M$ is $N$-function, we can choose $0<h<d$ such that $(1 / 2) M(d)+(1 / 2) M(h)-M((d+$ $h) / 2)>0$. Since $v_{n}(t) \rightarrow 0 \mu$ - a.e on $T$, by the Egorov theorem, there exists $N_{1}$ such that $\left\|v_{n}(t)\right\|<h, t \in F$ whenever $n>N_{1}$, where $F \subset T, \mu(T \backslash F)<(1 / 8) \mu T_{0}$. Next, we will prove that if $u_{1} \geq u_{2} \geq v_{2} \geq v_{1}>0$, then

$$
\frac{1}{2} M\left(u_{1}\right)+\frac{1}{2} M\left(v_{1}\right)-M\left(\frac{u_{1}+v_{1}}{2}\right) \geq \frac{1}{2} M\left(u_{2}\right)+\frac{1}{2} M\left(v_{2}\right)-M\left(\frac{u_{2}+v_{2}}{2}\right) .
$$

In fact, we have

$$
\begin{aligned}
\frac{1}{2} M\left(u_{1}\right)+\frac{1}{2} M\left(v_{1}\right)-M\left(\frac{u_{1}+v_{1}}{2}\right)-\left[\frac{1}{2} M\left(u_{1}\right)+\frac{1}{2} M\left(v_{2}\right)-M\left(\frac{u_{1}+v_{2}}{2}\right)\right] \\
=\frac{1}{2} M\left(v_{1}\right)-M\left(\frac{u_{1}+v_{1}}{2}\right)-\left[\frac{1}{2} M\left(v_{2}\right)-M\left(\frac{u_{1}+v_{2}}{2}\right)\right] \\
=\frac{1}{2} \int_{0}^{v_{1}} p(t) d t-\int_{0}^{\left(u_{1}+v_{1}\right) / 2} p(t) d t-\left[\frac{1}{2} \int_{0}^{v_{2}} p(t) d t-\int_{0}^{\left(u_{1}+v_{2}\right) / 2} p(t) d t\right] \\
=\left[\frac{1}{2} \int_{0}^{v_{1}} p(t) d t-\frac{1}{2} \int_{0}^{v_{2}} p(t) d t\right]+\left[\int_{0}^{\left(u_{1}+v_{2}\right) / 2} p(t) d t-\int_{0}^{\left(u_{1}+v_{1}\right) / 2} p(t) d t\right] \\
=-\frac{1}{2} \int_{v_{1}}^{v_{2}} p(t) d t+\int_{\left(u_{1}+v_{1}\right) / 2}^{\left(u_{1}+v_{2}\right) / 2} p(t) d t \\
\geq \int_{\left(u_{1}+v_{1}\right) / 2}^{\left(u_{1}+v_{2}\right) / 2} p(t) d t-\int_{\left(v_{1}+v_{2}\right) / 2}^{v_{2}} p(t) d t \geq 0 .
\end{aligned}
$$


Moreover, we have

$$
\begin{aligned}
\frac{1}{2} M\left(u_{1}\right)-M\left(\frac{u_{1}+v_{2}}{2}\right)-\left[\frac{1}{2} M\left(u_{2}\right)-M\left(\frac{u_{2}+v_{2}}{2}\right)\right] \\
\quad=\left[\frac{1}{2} \int_{0}^{u_{1}} p(t) d t-\frac{1}{2} \int_{0}^{u_{2}} p(t) d t\right]-\left[\int_{0}^{\left(u_{1}+v_{2}\right) / 2} p(t) d t-\int_{0}^{\left(u_{2}+v_{2}\right) / 2} p(t) d t\right] \\
\quad=\frac{1}{2} \int_{u_{2}}^{u_{1}} p(t) d t-\int_{\left(u_{2}+v_{2}\right) / 2}^{\left(u_{1}+v_{2}\right) / 2} p(t) d t \\
\geq \int_{u_{2}}^{\left(u_{1}+u_{2}\right) / 2} p(t) d t-\int_{\left(u_{2}+v_{2}\right) / 2}^{\left(u_{1}+v_{2}\right) / 2} p(t) d t \geq 0 .
\end{aligned}
$$

By (2.54) and (2.55), we have

$$
\frac{1}{2} M\left(u_{1}\right)+\frac{1}{2} M\left(v_{1}\right)-M\left(\frac{u_{1}+v_{1}}{2}\right) \geq \frac{1}{2} M\left(u_{2}\right)+\frac{1}{2} M\left(v_{2}\right)-M\left(\frac{u_{2}+v_{2}}{2}\right) .
$$

This shows that if $t \in T_{2}=T_{0} \backslash\left(T_{1} \cup(T \backslash F)\right)$, then

$$
M(\|u(t)\|)+M\left(\left\|v_{n}(t)\right\|\right)-2 M\left(\frac{\|u(t)\|+\left\|v_{n}(t)\right\|}{2}\right) \geq M(d)+M(h)-2 M\left(\frac{d+h}{2}\right) .
$$

It is easy to see $\mu T_{2} \geq(1 / 4) \mu T_{0}$. Therefore,

$$
\begin{aligned}
& \frac{1}{2} \rho_{M}(u)+\frac{1}{2} \rho_{M}\left(v_{n}\right)-\rho_{M}\left(\frac{u+v_{n}}{2}\right) \\
& \quad \frac{1}{2} \int_{T} M(\|u(t)\|) d t+\frac{1}{2} \int_{T} M\left(\left\|v_{n}(t)\right\|\right) d t-\int_{T} M\left(\frac{\left\|u(t)+v_{n}(t)\right\|}{2}\right) d t \\
& \quad \int_{T}\left[\frac{1}{2} M(\|u(t)\|)+\frac{1}{2} M\left(\left\|v_{n}(t)\right\|\right)-M\left(\frac{\left\|u(t)+v_{n}(t)\right\|}{2}\right)\right] d t \\
& \quad \geq \int_{T}\left[\frac{1}{2} M(\|u(t)\|)+\frac{1}{2} M\left(\left\|v_{n}(t)\right\|\right)-M\left(\frac{\|u(t)\|+\left\|v_{n}(t)\right\|}{2}\right)\right] d t \\
& \quad \geq \int_{T_{2}}\left[\frac{1}{2} M(\|u(t)\|)+\frac{1}{2} M\left(\left\|v_{n}(t)\right\|\right)-M\left(\frac{\|u(t)\|+\left\|v_{n}(t)\right\|}{2}\right)\right] d t \\
& \geq \int_{T_{2}}\left[\frac{1}{2} M(d)+\frac{1}{2} M(d)-M\left(\frac{d+h}{2}\right)\right] d t \\
& \geq\left[\frac{1}{2} M(d)+\frac{1}{2} M(d)-M\left(\frac{d+h}{2}\right)\right] \cdot \frac{1}{4} \mu T_{0},
\end{aligned}
$$

for $n$ large enough. By Lemma 1.1, we have $\rho_{M}(u)=\rho_{M}\left(v_{n}\right)=1, \rho_{M}\left(\left(u+v_{n}\right) / 2\right) \rightarrow 1$ as $n \rightarrow \infty$, which contradicts $(1 / 2) \rho_{M}(u)+(1 / 2) \rho_{M}\left(v_{n}\right)-\rho_{M}\left(\left(u+v_{n}\right) / 2\right) \geq[(1 / 2) M(d)+(1 / 2) M(d)-$ $M((d+h) / 2)] \cdot(1 / 4) \mu T_{0}$, for $n$ large enough. This completes the proof. 
Corollary 2.5. The following statements are equivalent:

(a) $L_{M}$ is locally uniformly nonsquare if and only if $L_{M}$ is nonsquare;

(b) $X$ is locally uniformly nonsquare.

\section{Acknowledgments}

The authors would like to thank the anonymous referee for some suggestions to improve the manuscript. This work was supported by China Natural Science Fund under Grant no. 11061022.

\section{References}

[1] R. C. James, "Uniform nonsquare Banach space," Annals of Mathematics, vol. 80, no. 3, pp. 542-550, 1964.

[2] J. García-Falset, E. Llorens-Fuster, and E. M. Mazcuñan-Navarro, “Uniformly nonsquare Banach spaces have the fixed point property for nonexpansive mappings," Journal of Functional Analysis, vol. 233, no. 2, pp. 494-514, 2006.

[3] S. T. Chen, "Geometry of Orlicz spaces," Dissertationes Math, vol. 356, pp. 1-204, 1996.

[4] C. X. Wu, T. F. Wang, S. T. Chen, and Y. W. Wang, Geometry Theory of Orlicz Spaces, H.I.T Print House, Harbin, China, 1986. 